\title{
Two are better than one: a design principle for ultralong persistent luminescence of pure organics
}

Parvej Alam ${ }^{1, \|}$, Nelson L. C. Leung ${ }^{1, \Perp}$, Junkai Liu ${ }^{1}$, Xuepeng Zhang ${ }^{1,2}$, Zikai He ${ }^{3}$, Ryan T. K. Kwok ${ }^{1,2}$, Jacky W. Y. Lam ${ }^{1,2}$, Herman H. Y. Sung ${ }^{1}$, Ian D. Williams ${ }^{1}$, Chris Chan ${ }^{4}$, Kam Sing Wong ${ }^{4}$, Qian Peng ${ }^{5}$, and Ben Zhong Tang $1,2,6 *$

${ }^{1}$ Department of Chemistry, the Hong Kong Branch of Chinese National Engineering Research Center for Tissue Restoration and Reconstruction and the Institute for Advanced Study, the Hong Kong University of Science and Technology, Clear Water Bay, Kowloon, Hong Kong, China

${ }^{2}$ HKUST-Shenzhen Research Institute, No. 9 Yuexing First Rd, South Area, Hi-Tech Park, Nanshan, Shenzhen 518057, China

${ }^{3}$ School of Science, Harbin Institute of Technology, Shenzhen, HIT Campus of University Town, 518055, Shenzhen, China

${ }^{4}$ Department of Physics, the Hong Kong University of Science and Technology, Clear Water Bay, Kowloon, Hong Kong, China

${ }^{5}$ Key Laboratory of Organic Solids, Beijing National Laboratory for Molecular Sciences, Institute of Chemistry, Chinese Academy of Sciences, Beijing 100190, China

${ }^{6}$ Centre for Aggregation-Induced Emission, SCUT-HKUST Joint Research Laboratory, State Key Laboratory of Luminescent Materials and Devices, South China University of Technology, Guangzhou 510640, China

\begin{abstract}
Because of their innate ability to store and then release energy, long persistent luminescence (LPL) materials have garnered strong research interest in a wide range of multidisciplinary fields, such as biomedical sciences ${ }^{1}$, theranostics ${ }^{2}$, and photonic devices ${ }^{3}$. Although many inorganic LPL systems with afterglow durations of up to hours and days have been reported ${ }^{3}$, organic systems have had difficulties reaching similar timescales ${ }^{4}$. We propose in this work a design principle based on the successes of inorganic systems to produce an organic LPL (OLPL) system through the use of a strong organic electron trap. The resulting system generated detectable afterglow for up to 7 hours, significantly longer than any other reported OLPL system ${ }^{5}$. The design strategy demonstrates an easy methodology to develop organic long persistent phosphors, opening the door to new OLPL materials.
\end{abstract}

Main

Long persistent luminescent ${ }^{6}(\mathrm{LPL})$ materials have demonstrated great potential and performance in multiple areas such as life sciences ${ }^{7}$, the biomedical field ${ }^{8}$, and photovoltaics ${ }^{9}$, as they offer fascinating possibilities for their 
ability to store and slowly release excited state energy. The most successful LPL materials make use of transition and rare-earth metal ions ${ }^{10,11}$. Although the metals grant exceptionally long afterglows, they are not without their inherent drawbacks. In addition to the high material costs of these rare-earth metals, many inorganic LPLs require harsh synthetic procedures ${ }^{3}$, further increasing research costs. LPL materials are also desirable for biomedical applications $^{12}$ for their ability to overcome autofluorescence. Organic LPL (OLPL) materials offer the promise of a multitude of benefits: easy synthesis, easy modification for targeted functionality, and easy processing. However, the development of OLPL materials have encountered many obstacles. To access long lived states in organic compounds, there have been many designs to exploit the excited triplet state. Though access to and from the triplet state is a forbidden process and once thought to be too inefficient for effective use at room temperature ${ }^{13}$, recent advances have vastly increased intersystem crossing efficiency by enhancing spin-orbit coupling (SOC) with the use of heteroatoms ${ }^{14,15}$, the carbonyl functional group ${ }^{16,17}$, heavy atom effects ${ }^{18}$, and multimer-enhance ISC $^{19}$. Equally important is protecting the long-lived triplet due to the fact that they are particularly sensitive to molecular vibrational quenching and atmospheric oxygen. In this regard, recent works have accomplished this through the use of crystals ${ }^{20,21}, \mathrm{MOFs}^{22}, \mathrm{H}$-aggregation ${ }^{23}$, and others ${ }^{24,25}$. Although there have been many achievements in generating organic room-temperature phosphorescent systems ${ }^{26-29}$, their lifetimes cannot match the durations of inorganic LPL materials.

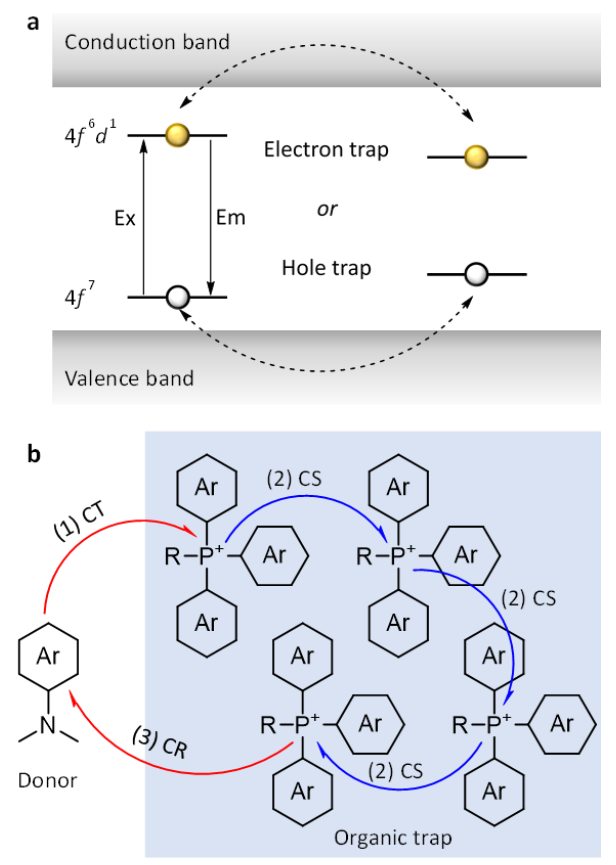

Figure 1 | Schematic representations of mechanisms behind inorganic and organic persistent luminescence.

a, Inorganic long persistent luminescence can be achieved through electron or hole trap mechanisms. In the electron trap, it is proposed after excitation (Ex), the excited electron travels through the conduction band to an electron accepting trap. In the hole trap, an electron fills the hole by traveling through the valence band. In both cases, relaxation becomes blocked, either because the excited electron has migrated away, or the hole has become filled. Thermal disturbances restore the electron or hole, producing afterglow emission (Em). b, A proposed mechanism for organic long persistent luminescence where a cationic quaternary phosphonium core acts as an organic trap and an aromatic amine as an electron donor. First (1) photoinduced charge transfer (CT) occurs 
between the donor and acceptor molecules followed by (2) charge separation (CS). Multiple CS can occur before the final step (3), charge recombination (CR), resulting in OLPL.

By applying concepts from the mechanistic understand of inorganic LPL systems to OLPLs, it can be possible to merge the diverse benefits of organic systems with the performance of inorganics. One of the most successful methods to achieve LPL in inorganic systems is through the use of traps (Fig. 1a), which allow for energy storage and its subsequent slow release via thermal disturbances. Inspired by the role of inorganic trap species, we hypothesized a purely organic bientitic system, a system made of two parts, i.e. i) an emitter and ii) a trap (Fig. 1b). Just as the heavy metals store charge carriers and slowly release them back into the system for eventual emission, organic molecules could theoretically do the same thing.

To test our mechanistic principle, we first set out to find an ionic core system that could work as a strong electron acceptor. In our search, we discovered, synthesized, and characterized (Fig. S1-5) a series of pure organic quaternary phosphonium bromide salts, TPP-3CB, TPP-4CB, TPP-3C2B, and TPP-4C2B, which exhibit observable afterglow (Fig. 2 and Supplementary Video 1-4). These four compounds were purified and recrystallized several times before characterization, with the ionic nature of the compounds aiding the formation of the crystal growth. Their crystal structures displayed a distorted tetrahedral geometry $\left(108.7^{\circ}\right.$ to $\left.110.8^{\circ}\right)$ and the distance between the phosphonium and bromide ions were measured to be in the range of $4.50 \AA$ to $4.75 \AA$ (Fig. S6). In all four phosphonium salts, none of them exhibit $\pi-\pi$ interactions or anionic bromide- $\pi$ interactions; examining their packing (Fig. S7) only $\mathrm{CH}^{\cdots} \pi$ and $\mathrm{CH}^{\cdots} \mathrm{Br}$ interactions exist. These interactions, as well as, electrostatic interactions work together to suppress molecular motion in the crystal enhancing phosphorescence efficiency. The absorption spectra of TPP-3CB, TPP-4CB, TPP-3C2B, and TPP-4C2B were recorded in dilute acetonitrile solutions $\left(1 \times 10^{-5} \mathrm{M}\right)$, and they showed similar absorption bands at $265 \mathrm{~nm}, 268 \mathrm{~nm}$, and $275 \mathrm{~nm}$ which were attributed to $\pi-\pi^{*}$ transitions from the phenyl moieties of the phosphonium core (Fig. S8). The crystals of all four phosphonium bromide salts showed an emission maximum of $480 \mathrm{~nm}$ (Fig. 2b), and surprisingly, their excitation spectra (Fig. S9) at $480 \mathrm{~nm}$ emission revealed a maximum excitation band at $310 \mathrm{~nm}$ which was attributed to a photoinduced charge transfer from the bromide counterion to the phosphonium core. Thus, subsequent PL and lifetime measurements of TPP-3CB, TPP-4CB, TPP-3C2B, and TPP-4C2B crystals were recorded by $310 \mathrm{~nm}$ excitation. From the time-resolved PL decay curves (Fig. 2c), the average lifetimes of TPP3CB, TPP-4CB, TPP-3C2B, and TPP-4C2B crystals at $480 \mathrm{~nm}$ measured at room temperature (298 K) were 157 ms, $200 \mathrm{~ms}, 159 \mathrm{~ms}$, and $164 \mathrm{~ms}$, respectively, indicating the ultralong afterglow nature of these materials and the average lifetimes of these phosphonium salts measured at $77 \mathrm{~K}$ in dilute solution were found to be $632 \mathrm{~ms}, 410$ ms, $320 \mathrm{~ms}$, and $717 \mathrm{~ms}$, respectively (Fig. S10-13). Under $254 \mathrm{~nm}$ hand held UV lamp excitation, intense greenish blue emission could be observed from the crystals (Fig. 2d). 
a

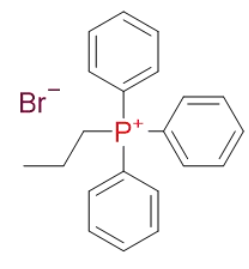

TPP-3CB

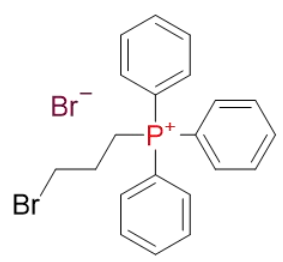

TPP-3C2B

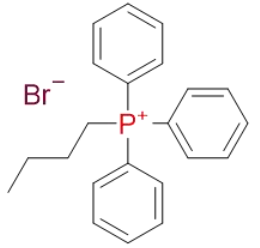

TPP-4CB

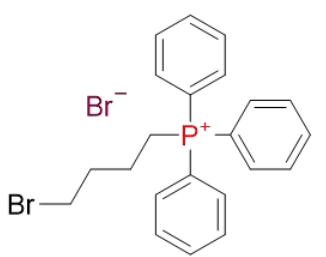

TPP-4C2B

C

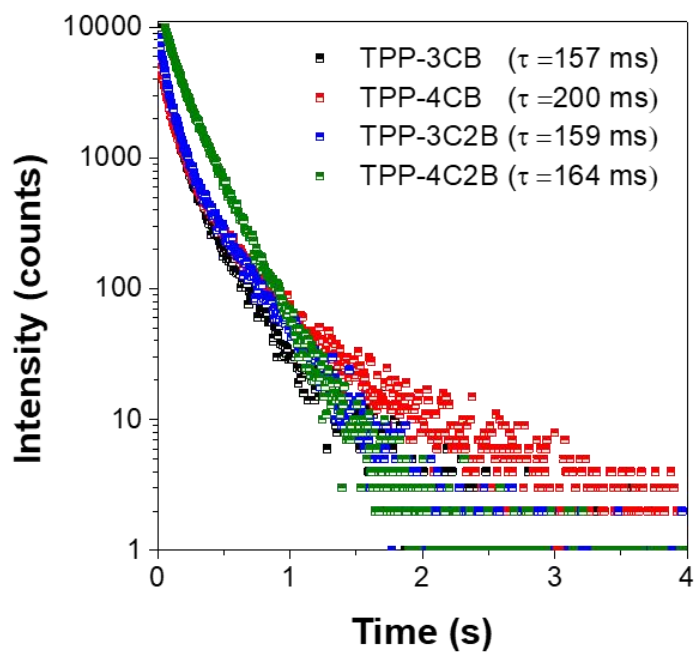

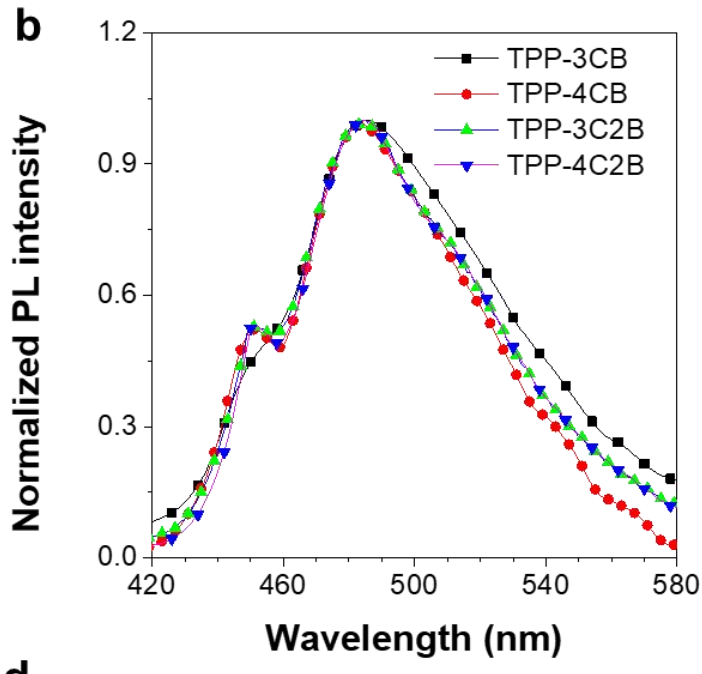

d
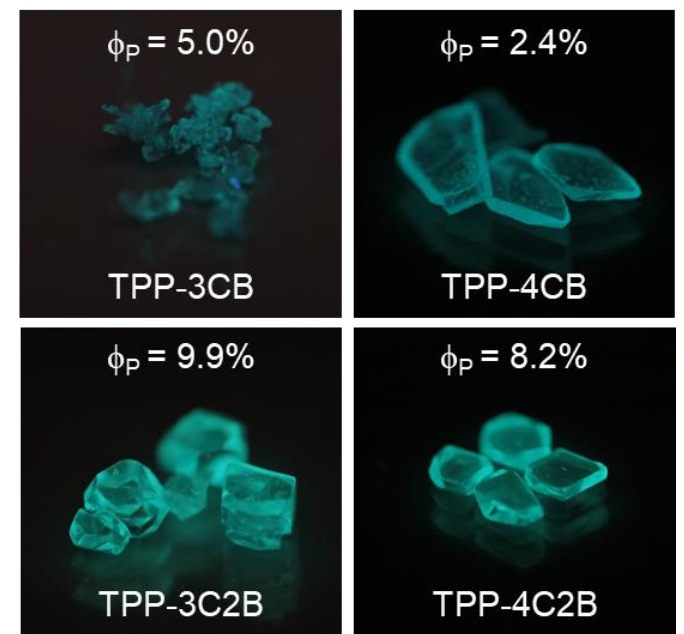

Figure 2 | Chemical structure and photophysical properties of phosphonium halides. a, Chemical structures, b, PL spectra and $\mathbf{c}$ time-resolved PL decay (at $480 \mathrm{~nm}$ ) of crystalline powders of TPP-3CB, TPP-4CB, TPP$3 \mathrm{C} 2 \mathrm{~B}$, and TPP-4C2B at $298 \mathrm{~K} ; \lambda_{\mathrm{ex}}=310 \mathrm{~nm}$. d, Photographs of TPP-3CB, TPP-4CB, TPP-3C2B, and TPP$4 \mathrm{C} 2 \mathrm{~B}$ under $\mathrm{UV}$ excitation at $254 \mathrm{~nm}$ as well as their phosphorescence quantum yields $\left(\Phi_{\mathrm{P}}\right)$.

We further took the crystal of TPP-3CB as a target model and employed combined quantum mechanics and molecular mechanics method (QM/MM) to simulate the photophysical process in the crystal, optimizing the excited singlet $\left(\mathrm{S}_{1}\right)$ and triplet $\left(\mathrm{T}_{1}\right)$ states and their molecular orbitals furnished by the ONIOM method (Fig. 3a$c$ and S14). At the $S_{1}$ geometry (Fig. 3a), the molecular orbitals of the $S_{1}$ and $T_{1}$ of TPP-3CB (Fig. S15) show complete charge transfer from its bromide anion to the phosphonium cation, furnishing excited radical pairs. Initially, a large separation of the orbitals involved in the transition from $S_{0}$ to $S_{1}$ resulted in a small exchange energy from $S_{1}$ to $T_{1}$ state, producing an energy gap between $S_{1}$ and $T_{1}$ that is smaller than $0.1 \mathrm{eV}$ facilitating intersystem crossing. Furthermore, the bromide anion endows the transition process with a spin-orbit coupling constant as large as $327.39 \mathrm{~cm}^{-1}$, which further enhances the transition between the $S_{1}$ and $T_{1}$ state. As such, both the complete charge-transfer characteristic and the strong SOC provides a firm foundation for the spin-orbit charge transfer (SOCT) intersystem crossing process from $S_{1}$ to $T_{1}$ for the phosphonium bromide complex. In addition, the excited state radical pair is also susceptible to hyperfine coupling (HFC) effects which provide another channel 
for intersystem crossing ${ }^{30}$; as the radicals are located on two separate locations, they experience different local magnetic fields leading to spin-mixing where rephasing (Fig. 3e) and spin-flipping transitions facilitate intersystem crossing. In this way, upon excitation the molecule initially becomes an excited singlet radical pair $\left({ }^{1} \mathrm{RP}\right)$, but due to SOCT and HFC effects, not only does the ${ }^{1} \mathrm{RP}$ easily intersystem cross to become an excited triplet radical pair $\left({ }^{3} \mathrm{RP}\right)$, the ${ }^{3} \mathrm{RP}$ can also easily intersystem cross back into the ${ }^{1} \mathrm{RP}$ (Fig. $3 \mathrm{~d}-\mathrm{e}$ ). According to the calculations, the RP state changes when the molecule relaxes to its $\mathrm{T}_{1}$ geometry, that is, at the optimized $\mathrm{T}_{1}$ geometry, charge-transfer/radical pair nature is lost. The molecular orbitals indicate at the $\mathrm{T}_{1}$ minimum, the exciton is localized on the phosphonium core (Fig. 3c) indicative of a second charge transfer event. The $\mathrm{T}_{1}$ becomes energetically separated from the $S_{1}$ with an energy difference of $0.957 \mathrm{eV}$ thus trapping the excited state for room-temperature phosphorescence.
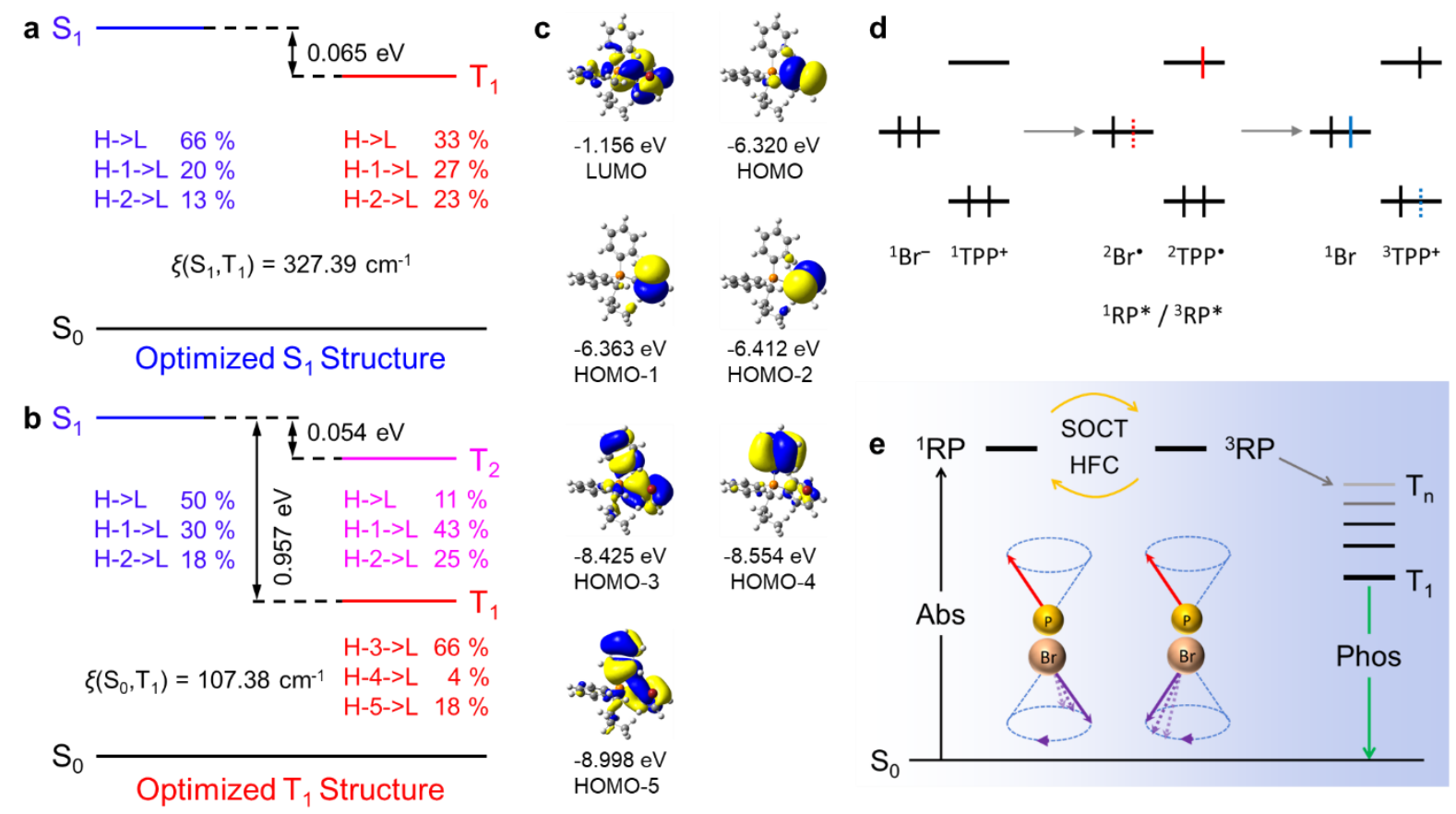

Figure 3 | QM/MM calculations at TD-DFT level of the singlet and triplet excited states. The TPP-3CB crystal structure was used to obtain an optimized geometry for the $\mathbf{a}, \mathrm{S}_{1}$ and $\mathbf{b}, \mathrm{T}_{1}$ excited state surrounded by 43 molecules. c, Molecular orbitals calculated from the optimized $\mathrm{T}_{1}$ structure. d, Schematic representation of electrons in TPP-3CB starting from the ground state, after the first charge transfer excitation (colored in red) into the radical pair (RP), and the second charge transfer event (colored in blue) into the $\mathrm{T}_{1}$ state. e, Modified Jablonski diagram showing the formation of the singlet radical pair $\left({ }^{1} \mathrm{RP}\right)$ after absorption (Abs). Spin-orbit charge transfer (SOCT) and hyperfine coupling (HFC) can aid in the interconversion between the ${ }^{1} \mathrm{RP}$ and the triplet radical pair $\left({ }^{3} \mathrm{RP}\right)$ as illustrated by the rephasing of the radical pair electrons in separate locations. The RP then relaxes into the $\mathrm{T}_{1}$ state before phosphorescence is observed.

Considering that it has been reported that excited-state charge separation can be beneficial for OLPLs ${ }^{5}$ and, taking cues from inorganic LPL systems, if we introduce moieties to "trap" charge carriers, it may be possible to further extend the afterglow duration. This could be made achieved by using a strong electron accepting molecule as the bulk. In other words, the bulk acts as one large "trap" slowing down recombination. To verify our hypothesis, we 
attempted to develop a bientitic OLPL system using a phosphonium bromide salt as a strong electron acceptor, which would act as the organic trap, and N,N-dimethylaniline (DMA) as an electron donor. We were able to dope DMA into TPP-3C2B by first mixing DMA with a dichloromethane (DCM) solution of TPP-3C2B and then allowing crystals to grow. These crystals exhibited a strong emission at $500 \mathrm{~nm}$ upon $365 \mathrm{~nm}$ excitation (Fig. 4a). For reference, the absorption spectrum of DMA in acetonitrile has two bands at $252 \mathrm{~nm}$ and $300 \mathrm{~nm}$ (Fig. S16) and has an emission band at $350 \mathrm{~nm}$. Since neither DMA nor TPP-3C2B have absorption bands at $365 \mathrm{~nm}$ (Fig. S17), this suggests the excitation of the TPP-3C2B:DMA crystals has photoinduced charge-transfer characteristics. Furthermore, the emission band of TPP-3C2B:DMA at $500 \mathrm{~nm}$ is $\sim 20 \mathrm{~nm}$ red-shifted from TPP3C2B, suggesting an exciplex emission (Fig. S18). The 2D excitation and emission maps of TPP-3C2B:DMA (Fig. S19a), TPP-3C2B (Fig. S19b), and DMA (Fig. S19c and d) further aid in the visualization. Pure DMA and TPP-3C2B show highly different maps when compared to TPP-3C2B:DMA. Specifically, at $365 \mathrm{~nm}$ excitation, neither TPP-3C2B nor DMA exhibit any detectable emission. In addition, the HPLC chromatograms (Fig. S20) confirm the presence of DMA in the TPP-3C2B:DMA samples. Furthermore, transient absorption of TPP3C2B:DMA detected the formation of a new band at $475 \mathrm{~nm}$ (Fig. S21). This matches well with the absorption of the $\mathrm{DMA}^{++}$produced in-situ in the presence of $\mathrm{Cu}\left(\mathrm{ClO}_{4}\right)_{2}$ (Fig. S21). After 60 seconds of $365 \mathrm{~nm}$ UV excitation, long-lived charge-separated states could be generated, and emission could be detected for up to $7 \mathrm{~h}$ (Fig. 4 and Supplementary Video 5) at ambient conditions.

Examining the emission decay profile under logarithmic x- and y-axes (Fig. S22), the profile matches a power decay which Hamill termed as Debye-Edwards law ${ }^{31}$, where PL intensity $\propto t^{-m}$. The Debye-Edwards law describes electron recombination kinetics where after excitation electrons become trapped in rigid media and slowly recombine with positive holes. The index $m$ reflects the rate of recombination and can have a numerical value from 0.1 to 2.0. The larger the $m$-value, the faster the decay. Typically, the exponent $m$ has a value $\sim 1^{5,10,31}$. In our system, fitting with the Debye-Edwards law produced an $m$-value of 0.716 , suggesting that the system has a significant impact to the emission decay characteristics. We believe the enhancement of the LPL duration is related to the positively charged phosphonium trap. The fitting further supports a charge transfer event and subsequent charge recombination process in the OLPL crystal.

The crystalline nature of TPP-3C2B:DMA protects the photo-generated radicals from atmospheric oxygen and suppresses nonradiative deactivation pathways allowing for such a long afterglow. These crystals exhibit good stability showing no visible changes to its afterglow duration even after being kept for more than 45 days under ambient dark conditions (Supplementary Video 6).

The reproducibility of this OLPL system was repeated several times by simply mixing of DMA/TPP-3C2B (10:1 mol ratio) in DCM/EA $(1: 1 \mathrm{v} / \mathrm{v})$ with varying maximum LPL durations ranging from 1 to $7 \mathrm{~h}$. We have also investigated how the DMA:TPP-3C2B ratio might affect the LPL duration. We grew crystals with 8-36 molar equivalent of DMA to TPP-3C2B (Fig. S23) and all the crystals grown exhibited 7 hours of LPL. The crystals grown from 8 and 12 molar equivalent of DMA exhibited slightly weaker emission at 7 hours when compared to the 16,18 , and 36 equivalents. The effect, however, does not appear to be particularly strong. We believe a key factor of the OLPL duration is the amount of DMA trapped inside the crystals. At this point in time, we are further investigating how to control this parameter. 


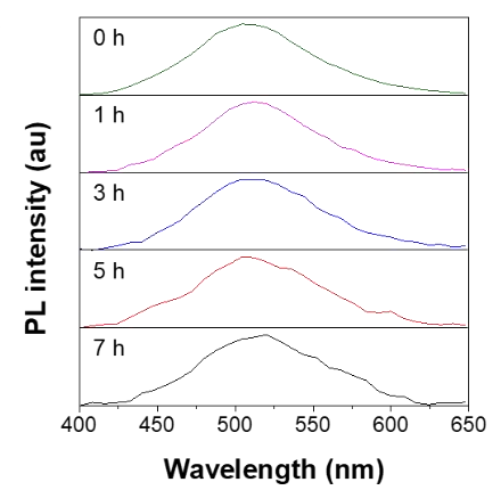

C

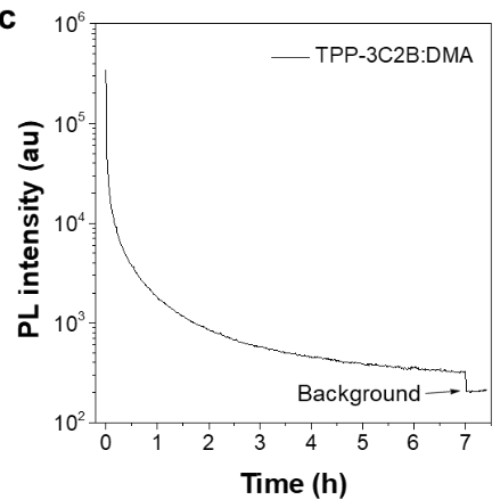

b
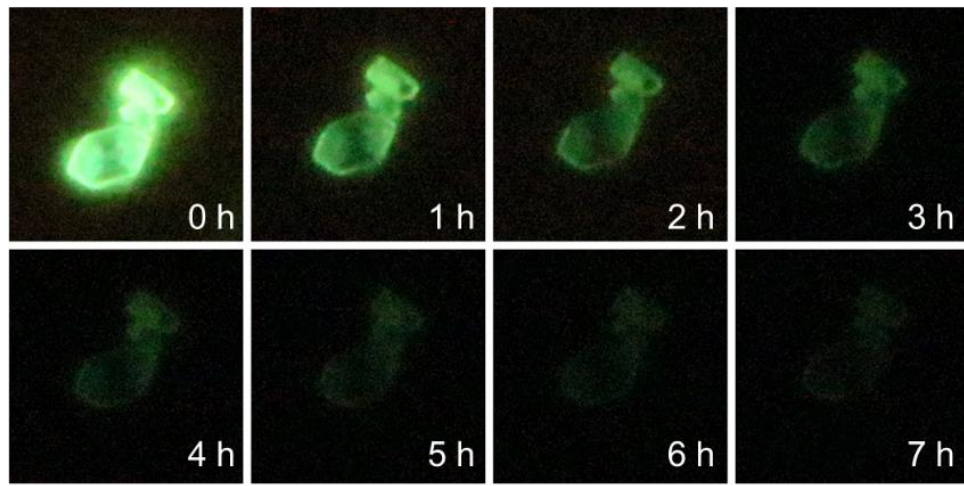

d
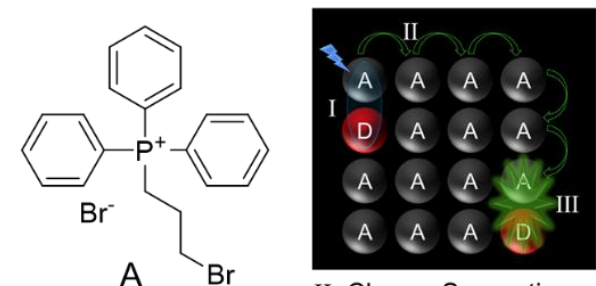

I. Charge Transfer
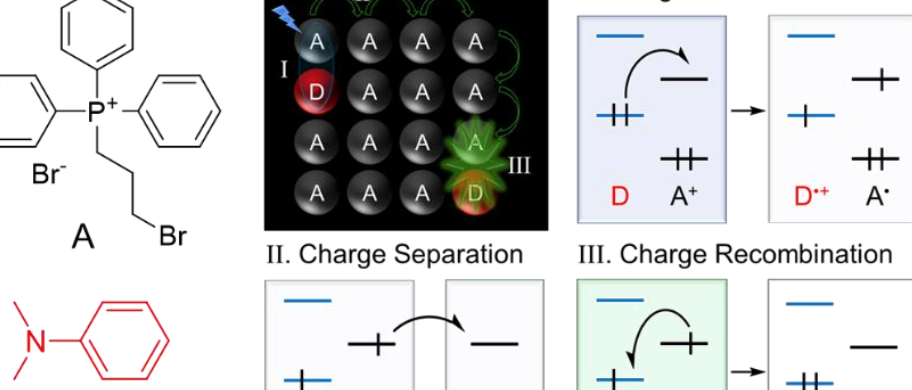

II. Charge Separation

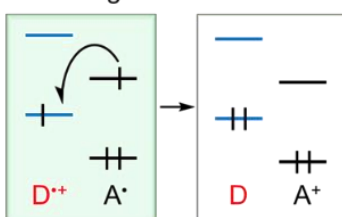

Figure 4 | Chemical structure and photophysical properties of TPP-3C2B:DMA. a, Emission spectra of TPP3C2B:DMA crystals measured at different times $(0 \mathrm{~h}$ to $7 \mathrm{~h})$ after the cessation of excitation at $365 \mathrm{~nm}$. b, Photographs of TPP-3C2B:DMA crystals taken under $365 \mathrm{~nm}$ UV excitation and its subsequent afterglow. c, Afterglow kinetic measurement of PL intensity at $500 \mathrm{~nm}$ over 7 hours. After 7 hours, the sample was removed to reveal background level measurement. d, Left: chemical structures of TPP-3C2B labelled (A) for acceptor, and N,N-dimethylaniline (DMA) labelled (D) for donor. Right: scheme representing the formation of the radical pair through charge transfer, subsequent charge separation and finally charge recombination to achieve long persistent luminescence.

Through careful analysis of the TPP-3C2B:DMA, it becomes possible to draw mechanistic insights to help design OLPL systems with even longer durations. The success of TPP-3C2B gives insight into factors that endow the system with such long persistent luminescence. One of the most important factors to the extraordinary duration of TPP-3C2B:DMA lies with the organic trap, the bulk crystal TPP-3C2B. Protection of the excited state is of utmost importance to ensuring high efficiency. In this case, the excited radical species, which are inherently reactive, can be easily lost. The phenyl rings decorating the phosphonium core stabilize the radical via steric protection ${ }^{32}$, acting as bulky barriers hindering unwanted reactions. In addition, calculations show the radical is able to delocalize amongst the phenyl rings (Fig. S24) further enhancing its stability ${ }^{32}$. The ionic nature of the material also aids in the crystallization, which protects the system from oxygen quenching maximizing emission efficiency. Another important factor that bestows such long durations is the trap's ability to hold onto the electron. The cationic nature of the phosphonium core is an ideal target to accept the electron of the photoinduced charge transfer process. Furthermore, being surrounded by other cationic phosphoniums, the excited radical can migrate to multiple cores 
(Fig. 4d), before finally recombining with a DMA radical resulting in the observed emission. Stronger, and better designed traps can likely even further increase the time the radical is stored before it jumps to another location. The principles exemplified by the success of TPP-3C2B can be applied to future OLPL systems producing even longer-lived emission and of varied colors and wavelengths. In addition, the OLPL system can be further tuned by modifying the donor molecule. Our group is currently working to develop OLPL systems based on these new insights.

In conclusion, we demonstrate a guiding design principle using strong organic traps to produce OLPL with previously unseen durations. The organic trap stabilizes and protects the excited radical, allowing the system to slowly recombine generating persistent luminescence. In TPP-3C2B:DMA, the cationic triphenylphosphonium core serves as a perfect trap, by first acting as a strong electron acceptor of the photoinduced charge transfer event, and then serving as multiple protective traps before the radical finally migrates back to a DMA for recombination. The insights demonstrated by our investigation will allow the future generation of highly persistent organic luminescent systems that may further propel the development and applications in the fields of medical science and optoelectronic devices.

\section{Methods}

All reagents were purchased from J\&K Scientific. HPLC grade solvents were purchased from Merck. All the molecules synthesized were purified by column chromatography and recrystallized using double layer solution diffusion with dichloromethane/hexane or dichloromethane/ethyl acetate for three times, and fully characterized by ${ }^{1} \mathrm{H}$ NMR, ${ }^{13} \mathrm{C}$ NMR, ${ }^{31} \mathrm{P}$ NMR, high-resolution mass spectroscopy (HRMS) and elemental analysis. ${ }^{1} \mathrm{H},{ }^{13} \mathrm{C}$, and ${ }^{31} \mathrm{P}$ NMR spectra were recorded on a Bruker AV 400 Spectrometer at 400, 100 and $162 \mathrm{MHz}$ in $\mathrm{CDCl}_{3}$, respectively. High-resolution mass spectra were recorded on a GCT premier CAB048 mass spectrometer operating in MALDI-TOF mode. The elemental analysis was performed on a Thermo Finnigan Flash EA1112. Gel filtration chromatography was performed using a ZORBAX SB-C18 column (Agilent) conjugated to an Agilent 1260 Infinite HPLC system. Before running, each sample was purified via a $0.22 \mu \mathrm{m}$ filter to remove any aggregates. The flow rate was fixed at $1.0 \mathrm{~mL} / \mathrm{min}$, the injection volume was $100 \mu \mathrm{L}$ and each sample was run for $10 \mathrm{~min}$. The absorption wavelength used was set at $254 \mathrm{~nm}$. Acetonitrile/water (95:5 v/v) was used as the running solvent system. The photoluminescence/excitation spectra were measured on a PerkinElmer LS 55 spectrophotometer and Horiba Fluoromax 4 spectrofluorometer. Quantum yields of the solids were recorded on Hamamatsu, C13534 at room temperature with a calibrated integrating sphere system. The lifetime was measured on an Edinburgh FLSP 920 fluorescence spectrophotometer equipped with a Xenon arc lamp (Xe900) and a microsecond flash-lamp (uF900). Single crystal data were collected on a Bruker Smart APEXII CCD diffractometer using graphite monochromated $\mathrm{Cu} \mathrm{K \alpha}$ radiation $(\lambda=1.54178 \AA$ ). The photos and videos were recorded by a Canon EOS 7D Mark II. The photographs for the OLPL were taken with 30-second-long exposures on a manual setting with f/2.8 and ISO 16000 to better capture the emission and were later joined together to form Supplementary Movie 5 and 6. The TD-DFT calculations were performed with the Gaussian 16 program.

\section{Data availability}


The X-ray crystallographic data for the structures of TPP-3CB, TPP-4CB, TPP-3C2B, and TPP-4C2B have been deposited at the Cambridge Crystallographic Data Centre (CCDC), under deposition numbers 1919500-1919503.

These data can be obtained free of charge from The Cambridge Crystallographic Data Centre via www.ccdc.cam.ac.uk/data_request/cif. Other data are available from the authors upon reasonable request.

\section{Acknowledgments}

This work was financially supported by the National Science Foundation of China (21788102), the Research Grants Council of Hong Kong (16308016, 16305518, C6009-17G and A-HKUST605/16), the Innovation and Technology Commission (ITC-CNERC14SC01 and ITC/254/17) and the Science and Technology Plan of Shenzhen (JCYJ20160229205601482 and JCYJ20170818113602462).

\section{Author information}

These authors contributed equally to this work: Parvej Alam, Nelson L. C. Leung.

\section{Affiliations}

Hong Kong Branch of Chinese National Engineering Research Center for Tissue Restoration and Reconstruction and Institute for Advanced Study, The Hong Kong University of Science and Technology, Clear Water Bay, Kowloon, Hong Kong, China

Parvej Alam, Nelson L. C. Leung, Junkai Liu, Xuepeng Zhang, Ryan T. K. Kwok, Jacky W. Y. Lam, Herman H. Y. Sung, Ian D. Williams, \& Ben Zhong Tang

HKUST-Shenzhen Research Institute, No. 9 Yuexing First Rd, South Area, Hi-Tech Park, Nanshan, Shenzhen 518057, China

Xuepeng Zhang, Ryan T. K. Kwok, Jacky W. Y. Lam, \& Ben Zhong Tang

School of Science, Harbin Institute of Technology, Shenzhen, HIT Campus of University Town, 518055, Shenzhen, China

Zikai He

Key Laboratory of Organic Solids, Beijing National Laboratory for Molecular Sciences, Institute of Chemistry, Chinese Academy of Sciences, Beijing 100190, China

Qian Peng

Centre for Aggregation-Induced Emission, SCUT-HKUST Joint Research Laboratory, State Key Laboratory of Luminescent Materials and Devices, South China University of Technology, Guangzhou 510640, China Ben Zhong Tang

\section{Contributions}

P.A., N.L.C.L., J.L., X.Z., Z.H., R.T.K.K., J.W.Y.L., and B.Z.T. conceived the experiments. P.A., N.L.C.L., and B.Z.T. prepared the paper. P.A. and N.L.C.L. were primarily responsible for the experiments. H.H.Y.S. and I.D.W. carried out the single crystal X-ray diffraction measurements and analyses. C.C and K.S.W. performed and analyzed the time-resolved absorption spectroscopy. J.L. and Q.P. contributed to the QM/MM TD-DFT calculations. All authors contributed to the data analyses.

\section{Competing interests}

The authors declare no competing interests. 


\section{Corresponding author}

Correspondence to Ben Zhong Tang (bztang@ust.hk).

\section{References}

1 le Masne de Chermont, Q. et al. Nanoprobes with near-infrared persistent luminescence for in vivo imaging. Proc. Natl. Acad. Sci. 104, 9266 (2007).

2 Liu, J. et al. Imaging and therapeutic applications of persistent luminescence nanomaterials. Adv. Drug Delivery Rev. 138, 193-210 (2019).

3 Li, Y., Gecevicius, M. \& Qiu, J. Long persistent phosphors-from fundamentals to applications. Chem. Soc. Rev. 45, 2090-2136 (2016).

$4 \quad \mathrm{Xu}, \mathrm{S}$., Chen, R., Zheng, C. \& Huang, W. Excited State Modulation for Organic Afterglow: Materials and Applications. Adv. Mater. 28, 9920-9940 (2016).

$5 \quad$ Kabe, R. \& Adachi, C. Organic long persistent luminescence. Nature 550, 384 (2017).

6 Harvey, E. N. A history of luminescence from the earliest times until 1900. Vol. 44 (American Philosophical Society, 1957).

7 Li, Z. et al. Direct Aqueous-Phase Synthesis of Sub-10 nm "Luminous Pearls" with Enhanced in Vivo Renewable Near-Infrared Persistent Luminescence. J. Am. Chem. Soc. 137, 5304-5307 (2015).

8 Maldiney, T. et al. The in vivo activation of persistent nanophosphors for optical imaging of vascularization, tumours and grafted cells. Nat. Mater. 13, 418 (2014).

9 Sun, H., Pan, L., Piao, X. \& Sun, Z. Enhanced performance of cadmium selenide quantum dotsensitized solar cells by incorporating long afterglow europium, dysprosium co-doped strontium aluminate phosphors. J. Colloid Interface Sci. 416, 81-85 (2014).

10 Pan, Z., Lu, Y.-Y. \& Liu, F. Sunlight-activated long-persistent luminescence in the nearinfrared from Cr3+-doped zinc gallogermanates. Nat. Mater. 11, 58 (2011).

11 Van den Eeckhout, K., Smet, P. F. \& Poelman, D. Persistent Luminescence in Eu(2+)-Doped Compounds: A Review. Materials 3, 2536-2566 (2010).

12 Smith, A. M., Mancini, M. C. \& Nie, S. Second window for in vivo imaging. Nat. Nanotechnol. 4, 710 (2009).

13 Wise, D. L., Wnek, G. E., Trantolo, D. J., Cooper, T. M. \& Gresser, J. D. Photonic Polymer Systems: Fundamentals: Methods, and Applications. (CRC Press, 1998).

14 Kenry, Chen, C. \& Liu, B. Enhancing the performance of pure organic room-temperature phosphorescent luminophores. Nat. Commun. 10, 2111 (2019).

15 Shoji, Y. et al. Unveiling a New Aspect of Simple Arylboronic Esters: Long-Lived RoomTemperature Phosphorescence from Heavy-Atom-Free Molecules. J. Am. Chem. Soc. 139, 2728-2733 (2017).

16 Zhao, W. et al. Rational Molecular Design for Achieving Persistent and Efficient Pure Organic Room-Temperature Phosphorescence. Chem 1, 592-602 (2016).

$17 \mathrm{Mu}, \mathrm{Y}$. et al. Mechano-induced persistent room-temperature phosphorescence from purely organic molecules. Chem. Sci. 9, 3782-3787 (2018).

18 Chen, G. et al. Photophysical Tuning of Organic Ionic Crystals from Ultralong Afterglow to Highly Efficient Phosphorescence by Variation of Halides. J. Phys. Chem. Lett. 9, 6305-6311 (2018).

19 Yang, J. et al. Elucidating the Excited State of Mechanoluminescence in Organic Luminogens with Room-Temperature Phosphorescence. Angew. Chem. Int. Ed. 56, 15299-15303 (2017).

20 Bolton, O., Lee, K., Kim, H.-J., Lin, K. Y. \& Kim, J. Activating efficient phosphorescence from purely organic materials by crystal design. Nat. Chem. 3, 205 (2011).

21 Yuan, W. Z. et al. Crystallization-Induced Phosphorescence of Pure Organic Luminogens at Room Temperature. J. Phys. Chem. C 114, 6090-6099 (2010).

22 Yang, X. \& Yan, D. Strongly Enhanced Long-Lived Persistent Room Temperature Phosphorescence Based on the Formation of Metal-Organic Hybrids. Adv. Opt. Mater. 4, 897905 (2016). 
23 An, Z. et al. Stabilizing triplet excited states for ultralong organic phosphorescence. Nat. Mater. 14, 685 (2015).

24 Hirata, S. Recent Advances in Materials with Room-Temperature Phosphorescence: Photophysics for Triplet Exciton Stabilization. Adv. Opt. Mater. 5, 1700116 (2017).

25 Forni, A., Lucenti, E., Botta, C. \& Cariati, E. Metal free room temperature phosphorescence from molecular self-interactions in the solid state. J. Mater. Chem. C 6, 4603-4626 (2018).

26 Chen, H., Ma, X., Wu, S. \& Tian, H. A Rapidly Self-Healing Supramolecular Polymer Hydrogel with Photostimulated Room-Temperature Phosphorescence Responsiveness. Angew. Chem. Int. Ed. 53, 14149-14152 (2014).

27 Mukherjee, S. \& Thilagar, P. Recent advances in purely organic phosphorescent materials. Chem. Commun. 51, 10988-11003 (2015).

28 Yang, Z. et al. Intermolecular Electronic Coupling of Organic Units for Efficient Persistent Room-Temperature Phosphorescence. Angew. Chem. Int. Ed. 55, 2181-2185 (2016).

29 Zhang, G., Palmer, G. M., Dewhirst, M. W. \& Fraser, C. L. A dual-emissive-materials design concept enables tumour hypoxia imaging. Nat. Mater. 8, 747 (2009).

30 Turro, N. J. Modern molecular photochemistry. (University science books, 1991).

31 Hamill, W. H. Debye-Edwards electron recombination kinetics. J. Chem. Phys. 71, 140-142 (1979).

32 Tidwell, T. T. in Stable Radicals: Fundamentals and Applied Aspects of Odd-Electron Compounds (ed Robin G. Hicks) Ch. 1, 1-31 (Wiley, 2010). 